\title{
Uso de Vinaza de Saccharum officinarum para Estabilización de Suelos Cohesivos
}

\section{Use of Saccharum officinarum Vinasse for Stabilization of Cohesive Soils}

Flor Lisbet Bustamante Salazar

Escuela profesional de Ingeniería Civil

Universidad César Vallejo

Trujillo, Perú

bsalazafl@ucvvirtual.edu.pe
Noé Humberto Marín Bardales

Escuela profesional de Ingeniería Civil Universidad César Vallejo

Trujillo, Perú

mbardalesn@ucvvirtual.edu.pe
Julio César Benites Chero

Escuela profesional de Ingeniería Civil

Universidad César Vallejo

Trujillo, Perú

jbenitesce@ucvvirtual.edu.pe

Fecha de recepción: 28/06/2021 - Fecha de aprobación: 30/11/2021

\section{RESUMEN}

El presente artículo muestra el uso de la vinaza de Saccharum officinarum en la estabilización de suelos cohesivos. Estos suelos en mención son aquellos que presentan propiedades de resistencia muy pobres, lo cual impide que el suelo trabaje de una forma adecuada como parte de una subrasante en una vía. El objetivo de la investigación fue determinar la estabilización de suelos cohesivos con la incorporación de vinaza de Saccharum officinarum. El tipo de investigación es experimental, la técnica utilizada es la observación y ficha de recolección de datos, mediante en la cual se pudo obtener valores de los ensayos realizados en laboratorio con el suelo natural y suelo estabilizado con vinaza de Saccharum officinarum. Los resultados indican que el suelo en estudio son limos y arcillas inorgánicas de baja plasticidad. Con la aplicación de vinaza de Saccharum officinarum se identificó que la densidad del suelo aumenta con los diferentes porcentajes utilizados. Luego al realizar los ensayos de resistencia mecánica del suelo estabilizado se logró incrementar la resistencia respecto a la del suelo natural, siendo el $25 \%$ de vinaza de Saccharum officinarum la que obtuvo los valores más altos. La investigación concluye que la incorporación de vinaza de Saccharum officinarum aumenta las propiedades de resistencia de un suelo cohesivo.

Palabras clave: suelos cohesivos, estabilización de suelos, vinaza de Saccharum officinarum, resistencia.

\section{ABSTRACT}

This article shows the use of Saccharum officinarum stillage in stabilizing cohesive soils. These soils in mention are those that present very poor resistance properties, which prevents the soil from working properly as part of a subgrade on a road. The objective of the research was to determine the stabilization of cohesive soils with the incorporation of vinasse from Saccharum officinarum. The type of research is experimental, the technique used is observation and data collection sheet, through which it was possible to obtain values from the tests carried out in the laboratory with natural soil and soil stabilized with Saccharum officinarum vinasse. The results indicate that the soil under study is low plasticity inorganic silts and clays. With the application of Saccharum officinarum vinasse, it was identified that the density of the soil increases with the different percentages used. Then, when carrying out the mechanical resistance tests of the stabilized soil, it was possible to increase the resistance with respect to that of the natural soil, being $25 \%$ of Saccharum officinarum vinasse the one that obtained the highest values. Research concludes that the incorporation of Saccharum officinarum stillage increases the strength properties of a cohesive soil.

Keywords: cohesive soils, soil stabilization, Saccharum officinarum vinasse, strength 


\section{Introducción}

La estabilización de suelos hoy en día es de suma importancia ya que ayuda a mejorar las características geotécnicas de un suelo pobre y así usarlo para la construcción de diversas obras civiles. Para ello, se han utilizado diversos productos en la estabilización de suelos cohesivos, desde productos de reconocida trayectoria en el mundo de la construcción, hasta el uso de productos alternativos, bajo el contexto de cuidado ambiental y reutilización de ciertos materiales. Es por ello que se hace referencia a la vinaza de Saccharum officinarum como el efluente o líquido residual de la producción de alcohol a partir de la fermentación del jugo extraído de la fibra de Saccharum officinarum después de su aplastamiento en las máquinas. Se caracteriza por tener sólidos suspendidos, color marrón oscuro o vino tinto con olor a miel (Alzate, 2015).

Luego, Amunza (2020) en su estudio propuso determinar el mejor porcentaje de desempeño de estabilización con melaza de caña de azúcar, llegando a la conclusión que el porcentaje óptimo fue el $2 \%$. Así mismo, Kiran y colaboradores (2018), propusieron como objetivo la incorporación del 4\%,6\%, 8\% y $10 \%$ de melaza y el del $2 \%, 4 \%, 6 \%, 8 \%, 10 \%$ y $12 \%$ de fibra de caña. Llegaron a determinar que el mejor aglomerante es la melaza con la adición del 8\%.

Luego, Lozano y colaboradores (2015) en su investigación propusieron determinar el efecto de la incorporación del aditivo orgánico en el suelo seleccionado para mejorar. Los autores llegaron a la conclusión que al adicionar el $7 \%$ del aglomerante el suelo muestra valores elevados en cuanto a su capacidad de resistencia con un valor de $12,8 \%$ con respecto al Californian Bearing Ratio (CBR).

A la vez, Bhardwaj y colaboradores (2021) en su artículo plantearon mejorar las propiedades geotécnicas del suelo con la incorporación de melaza de caña de azúcar y melaza; llegando a concluir que la adición de melaza de caña de azúcar aumenta la máxima densidad seca y disminuye el contenido de humedad.

En la actualidad, las trochas carrozables muestran un bajo soporte del suelo, lo cual no garantiza una estabilidad del suelo de la vía. El principal problema de una trocha carrozable o caminos rurales es la estabilización inadecuada. En muchos casos, no se realizan estudios previos para verificar la resistencia de la subrasante.

\section{Objetivos}

Los objetivos de la investigación fueron:

Reconocer las características geotécnicas del suelo cohesivo; aplicar la vinaza de Saccharum officinarum mediante porcentajes de $10 \%, 15 \%, 20 \%$ y $25 \%$ en el suelo cohesivo; plasmar las propiedades mecánicas de la estabilización del suelo cohesivo con la incorporación de vinaza de Sacccharum officinarum y determinar la dosificación adecuada de incorporación de la vinaza al suelo cohesivo.

\section{Metodología}

La metodología que desarrolló la investigación estuvo dividida en tres fases, ello se muestra en la Figura 1. La Fase I (Revisión bibliográfica) contempló los aspectos relacionados con antecedentes de investigación, los objetivos del estudio, la normativa y la selección de los porcentajes de aplicación de la vinaza de Saccharum officinarum. La Fase II (Desarrollo experimental) contempló la extracción de muestras del suelo cohesivo, la adición de los porcentajes de vinaza de Saccharum officinarum y los ensayos de laboratorio.

La extracción de muestras de suelo cohesivo siguió los parámetros de exploración de la normativa del Ministerio de Transporte y Comunicaciones del Perú (2017).

Los ensayos de laboratorio están referidos al análisis granulométrico por tamizado (MTC E107, R204/ ASTM D 422/ AASTHO T-11, T-27, T-88), límites de Atterberg (MTC E10, E 11/ ASTM D 4318/AAST-89, T-90) y gravedad específica (ASTM D854) para determinar sus características geotécnicas. Luego, para determinar sus propiedades de máxima densidad seca y valor de soporte del suelo natural se utilizó el ensayo de Proctor Modificado (MTC E 115 /ASTM D 1557) y la determinación del valor de soporte (CBR) (MTC E132/ ASTM D1883 /AASTHO T-193), respectivamente. 
Además, en la misma Fase II, se adiciona la vinaza de Saccharum officinarum en porcentajes de 10\%, 15\%, $20 \%$ y $25 \%$ del peso de la muestra de suelo cohesivo. Luego, se procedió a determinar los valores de su máxima densidad y valor de soporte con los ensayos mencionados en dicha fase.
La Fase III (Análisis de datos) abarcó la discusión de los resultados (análisis y comparación de resultados), conclusiones y recomendaciones.

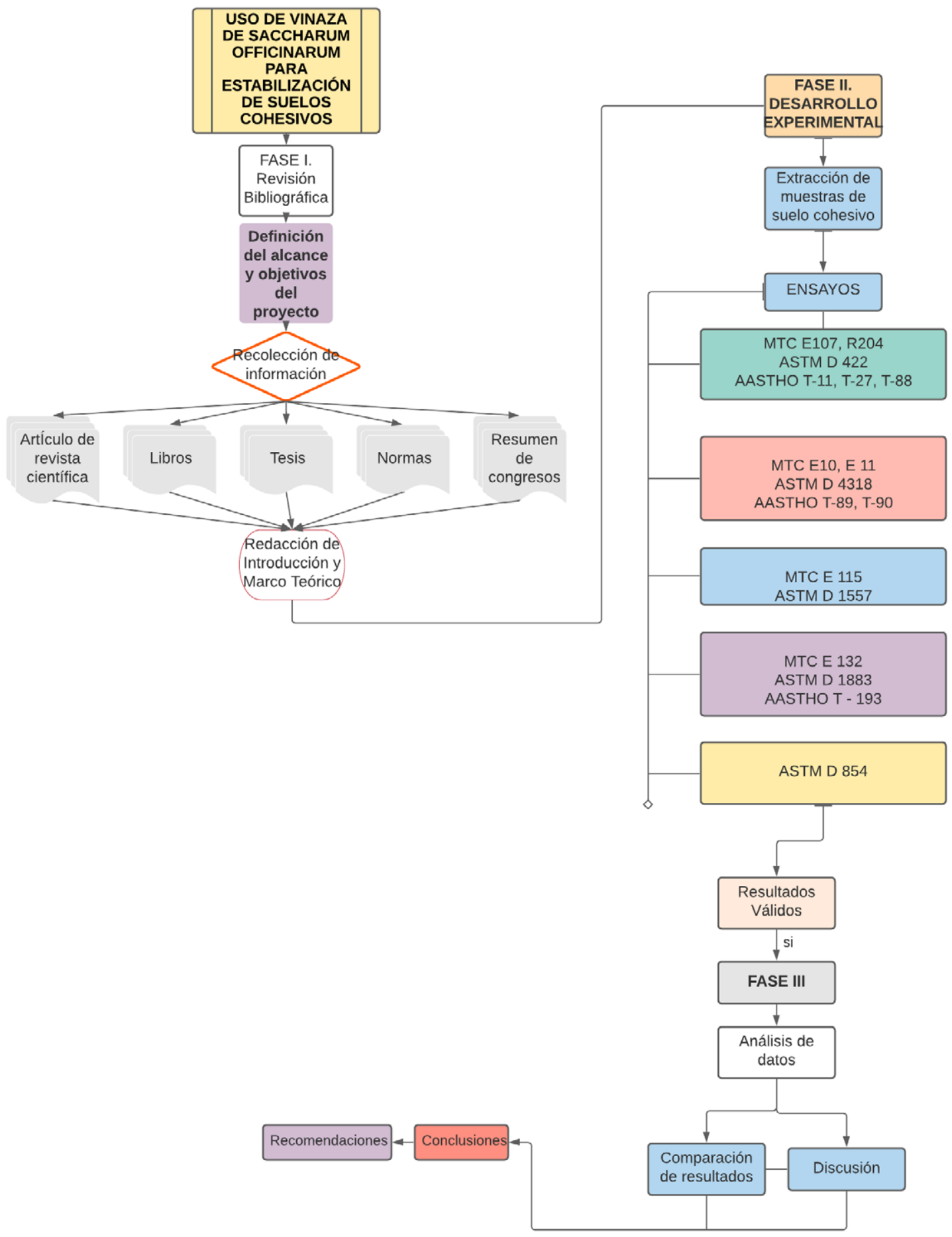

Figura 1. Diagrama de metodología aplicada 
La Figura 2 plasma la extracción de muestras de suelo cohesivo. La Figura 3 y la Figura 4 evidencia los ensayos de laboratorio a los cuales fue sometida la muestra de suelo cohesivo como son los límites de Atterberg (MTC E10, E 11/ ASTM D 4318/AAST-89,T-90) y determinación del valor de soporte (CBR) (MTC E132/ ASTM D1883 /AASTHO T-193), respectivamente.

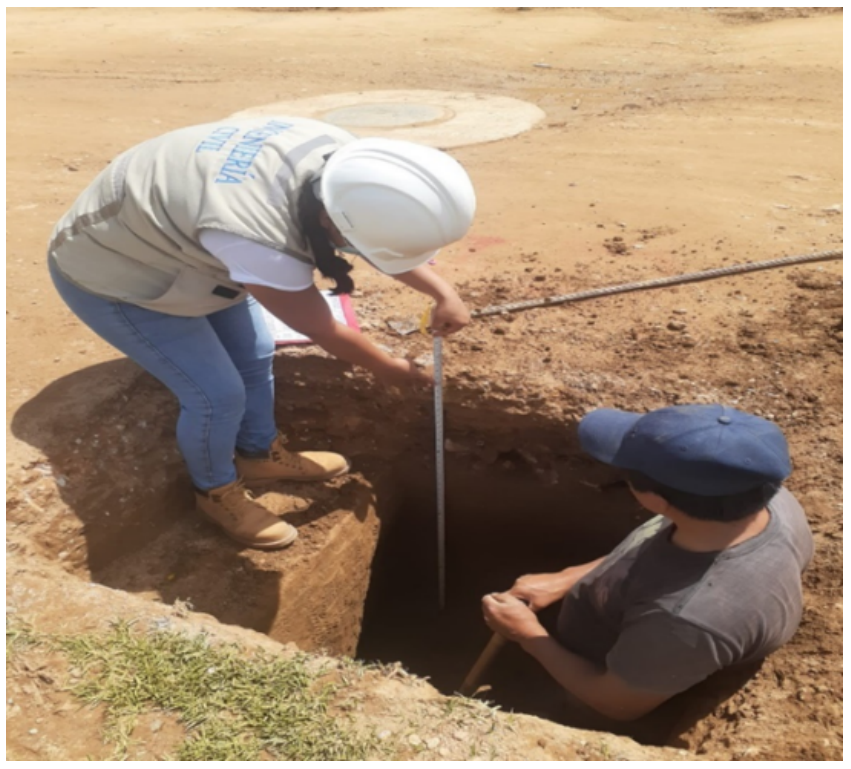

Figura 2. Extracción de muestra de suelo (Fase II)

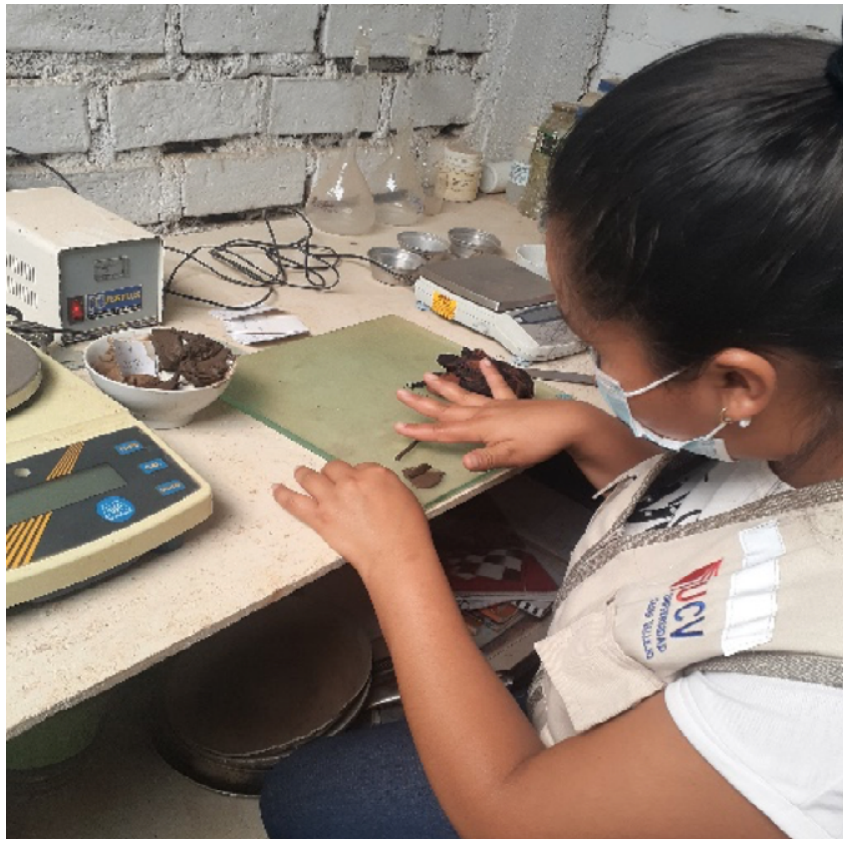

Figura 3. Ensayos de laboratorio de las muestras de suelo (Fase II)

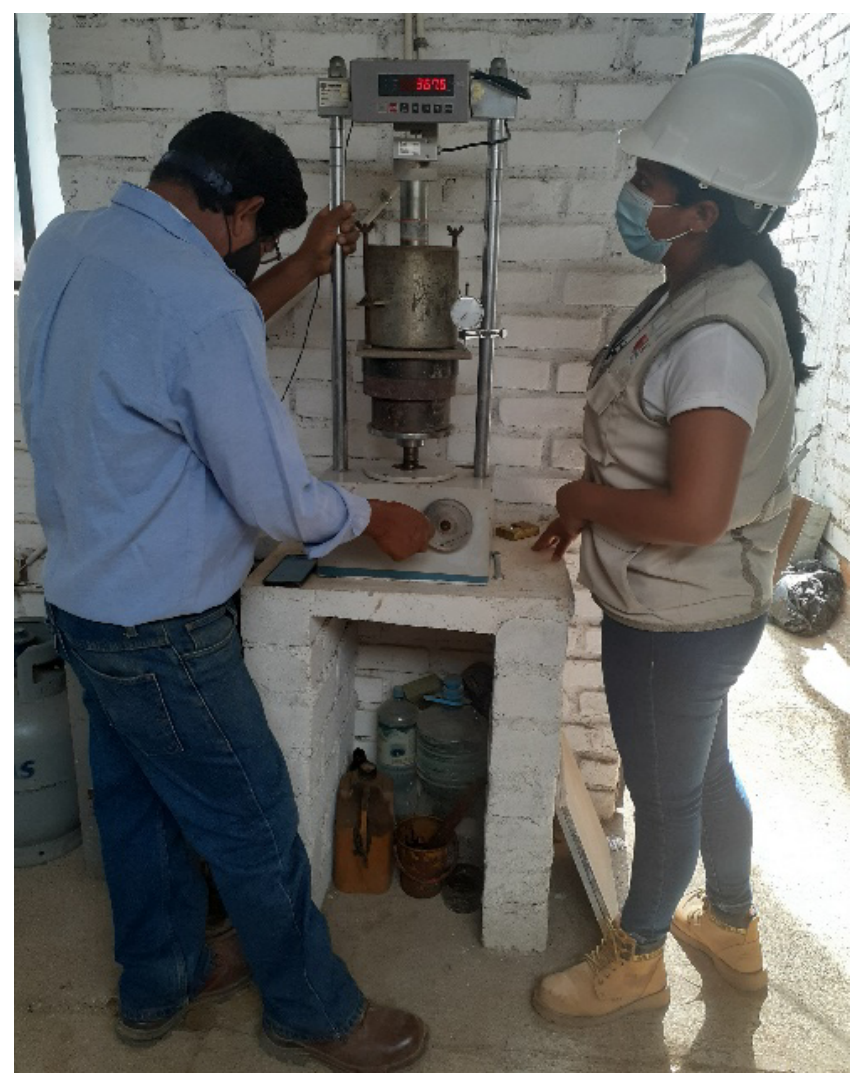

Figura 4. Ensayo del Valor de soporte de California (CBR) del suelo cohesivo con la vinaza (Fase II)

\section{Ensayo de análisis granulométrico}

El ensayo consiste en la clasificación de la muestra del suelo según el tamaño de sus partículas. Este ensayo se elabora para determinar el tipo de suelos que se encuentra en una muestra, verificando los porcentajes de muestra retenidos y los porcentajes que pasan en cada tamiz (Ministerio de Transportes y Comunicaciones, 2017).

\section{Límite Plástico}

Este ensayo se define como la representación de la humedad más baja del suelo. El ensayo de límite plástico se realiza para determinar las fracciones de material fino de una muestra con la cual se forma cilindros de $3 \mathrm{~mm}$ de diámetro (Figura 3), los cuales se ruedan en una superficie plana hasta lograr que los cilindros antes realizados muestren grietas. Luego, los cilindros son llevados al horno para obtener el máximo porcentaje de humedad (Ministerio de Transportes y Comunicaciones, 2017). 


\section{Límite líquido (L.L)}

El L.L del suelo contiene gran cantidad de materia orgánica, la cual disminuye cuando la muestra del suelo es secada en el horno. Este ensayo se realiza para la obtención del contenido de humedad por debajo del cual la muestra del suelo se comporta como material plástico (Ministerio de Transportes y Comunicaciones, 2017).

\section{Proctor modificado}

El ensayo en el cual se busca mejorar las propiedades de resistencia, compresión y esfuerzo deformación del suelo. El proceso de este ensayo implica la reducción de vacíos dentro de la muestra del suelo sin dañar su estructura (Ministerio de Transportes y Comunicaciones, 2017).

\section{CBR (Californian Bearing Ratio)}

En la Figura 4 se muestra la ejecución del ensayo, el cual se realiza para determinar el índice de resistencia del suelo, denominado valor de soporte. El método del CBR es usado para la evaluación de resistencia de subrasante, subbase y base del suelo (Ministerio de Transportes y Comunicaciones, 2017).

\section{Peso específico}

Es la relación entre peso y volumen, lo cual hace que el valor sea dependiente de la humedad, del peso específico de las partículas del suelo y de los vacíos de aire (Ministerio de Transportes y Comunicaciones, 2017).

\section{Aplicación de la vinaza de Saccharum officinarum}

La vinaza es un producto de desecho que resulta del proceso de producción del azúcar en las plantas agroindustriales. Se constituye por nitrógeno, azufre, fósforo y potasio; su color característico es marrón oscuro y de textura densa (Zúñiga y Gandini, 2013).

Sobre la aplicación en laboratorio de la vinaza de Saccharum officinarum en el suelo cohesivo se selecionaron los porcentajes propuestos por los investigadores Cahuana (2018) y Cordova (2018), los cuales se muestran en el Cuadro 1.
Cuadro 1. Peso de vinaza Saccharum

officinarum aplicado al suelo natural

\begin{tabular}{|c|c|}
\hline $\begin{array}{c}\text { Vinaza Saccharum } \\
\text { officinarum (\%) }\end{array}$ & $\begin{array}{c}\text { Peso utilizado } \\
\text { (gramos) }\end{array}$ \\
\hline 10 & 50,00 \\
\hline 15 & 75,00 \\
\hline 20 & 100,00 \\
\hline 25 & 125,00 \\
\hline
\end{tabular}

La Fase III consistió en el análisis de resultados, discusión y conclusiones de la investigación

\section{Análisis de resultados}

Al reconocer las características geotécnicas del suelo cohesivo (Cuadro 2), se encontró dos tipos de suelo según SUCS (Sistema Unificado de Clasificación de Suelos). Las calicatas 1, 2 y 4 muestran un suelo limoso inorgánico de mediana plasticidad, de consistencia ligeramente compactada en estado húmedo (ML). La C3 muestra un suelo arcilloso inorgánico de mediana plasticidad (CL).

La investigación de Amunza (2020) menciona que el ensayo de granulometría brindó la clasificación del suelo, el cual lo clasifica como grava bien graduada según el Sistema Unificado de Clasificación de Suelos. De la misma forma, Becerra (2019) realizó el ensayo de límite líquido obteniendo como resultado 11,8\% y límite plástico $0 \%$, donde obtuvo un suelo gravoso con arcillas y arenas.

Estas investigaciones concuerdan con los ensayos que se realizaron en el presente estudio, ya que ambas siguen el mismo proceso para obtener sus resultados. Las muestras del suelo encontrado se clasifican como un limo inorgánico (ML) y una arcilla inorgánica de baja plasticidad (CL). Los límites de Atterberg expresan que las muestran de suelos presentan en su mayoría altos índices de plasticidad superior a $15 \%$.

La aplicación de porcentajes de vinaza de Saccharum officinarum (VSO) se muestra en la Figura 5 . En el Cuadro 3 se identificó que el peso de la muestra natural aumenta con respecto a la cantidad de vinaza adicionada. 


\section{Cuadro 2. Características geotécnicas del suelo cohesivo natural}

\begin{tabular}{|c|c|c|c|c|}
\hline Grupos & \multicolumn{3}{|c|}{ Grupo 1 } & Grupo 2 \\
\hline Calicatas & $\mathrm{C} 1$ & $\mathrm{C} 2$ & $\mathrm{C} 4$ & $\mathrm{C} 3$ \\
\hline SUCS & $\mathrm{ML}$ & $\mathrm{ML}$ & $\mathrm{ML}$ & $\mathrm{CL}$ \\
\hline AASHTO & $\mathrm{A}-7-6(11)$ & $\mathrm{A}-7-68(13)$ & $\mathrm{A}-6(10)$ & $\mathrm{A}-7-5(13)$ \\
\hline Límite Líquido & 41,4 & 46,2 & 37,9 & 48,8 \\
\hline Límite plástico & 25,9 & 27,3 & 23,5 & 30,7 \\
\hline Índice de plasticidad & 15,5 & 18,9 & 7,8 & 18,1 \\
\hline Contenido de humedad & $32,3 \%$ & $24,9 \%$ & $29,2 \%$ & $35,4 \%$ \\
\hline Peso específico & 2,439 & 2,414 & 2,51 & 2,414 \\
\hline
\end{tabular}

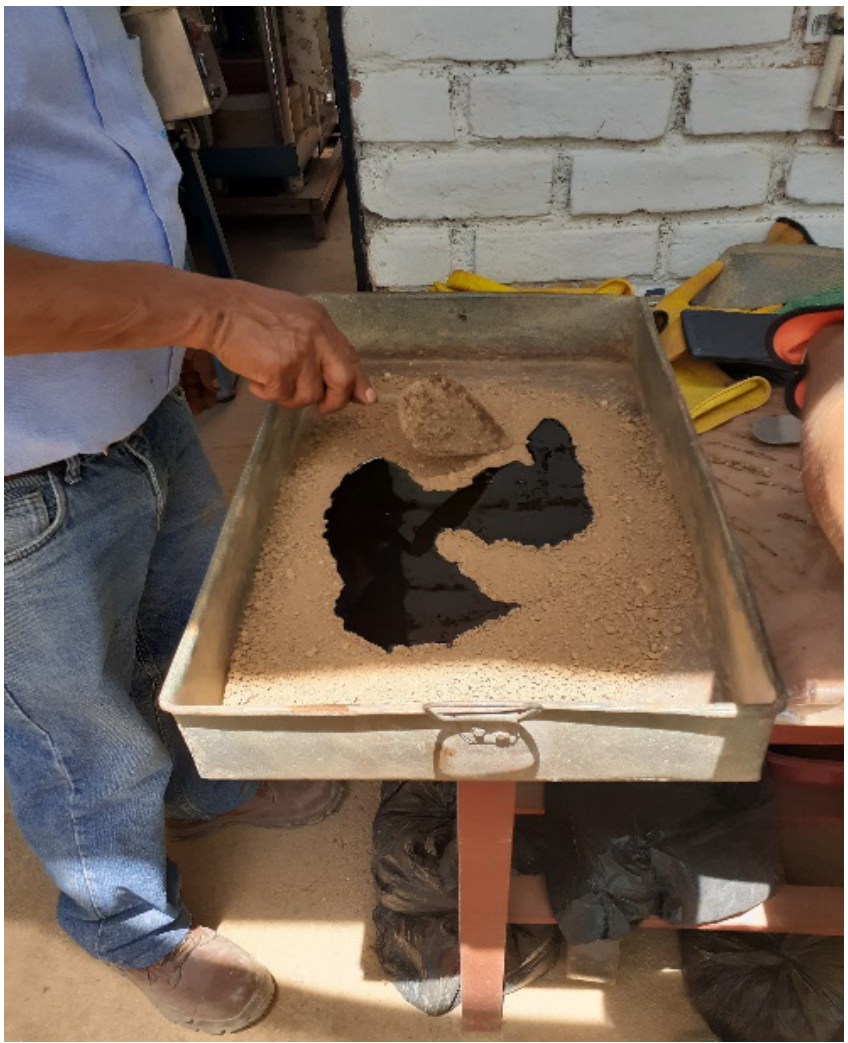

Figura 5. Aplicación de la vinaza de Saccharum officinarum a la muestra de suelo
Kiran et al., (2018) en su investigación hicieron mención de que el suelo natural de los cultivos de algodón negro presenta altos contenidos de arcillas. Por lo cual, propusieron realizar una estabilización de suelos con los siguientes porcentajes: $4 \%, 6 \%$, $8 \%$ y $10 \%$ de vinaza Saccharum officinarum del peso de la muestra del suelo cohesivo. Esta investigación presenta la misma metodología del estudio realizado en utilizar diferentes porcentajes del peso de la muestra de suelo cohesivo.

En la Figura 6 se muestran los resultados del ensayo Proctor modificado de la C2 para determinar las propiedades mecánicas del suelo cohesivo mediante la incorporación de los diferentes porcentajes $(10 \%, 15 \%, 20 \%$ y $25 \%)$ de vinaza de Saccharum officinarum.

En la Figura 7 se muestran los resultados del ensayo de CBR al 95\% donde se aprecia el aumento de la resistencia del suelo con respecto al suelo natural, un CBR que oscila entre $9,3 \%$ y $6,9 \%$ con la incorporación del 10\% de VSO; un CBR con resultados entre 11,3\% y $8,4 \%$ con la incorporación del $15 \%$ de VSO; un CBR con resultados entre $13,5 \%$ y $9,9 \%$ con el $20 \%$ de VSO y, por último, un CBR el cual oscila entre 15,8\% y $11,8 \%$ con la incorporación del $25 \%$ de VSO.

Cuadro 3. Aplicación de porcentajes de vinaza de Saccharum officinarum (VSO), según peso de muestra

\begin{tabular}{|c|c|c|c|c|c|}
\hline \multirow{2}{*}{ Calicata } & \multirow{2}{*}{ Peso de muestra } & \multicolumn{4}{|c|}{ Peso de VSO } \\
\cline { 3 - 6 } & & $10 \%$ VSO & $15 \%$ VSO & $20 \%$ VSO & $25 \%$ VSO \\
\hline C1 & $500,00 \mathrm{~g}$ & $50,00 \mathrm{~g}$ & $75,00 \mathrm{~g}$ & $100,00 \mathrm{~g}$ & $125,00 \mathrm{~g}$ \\
\hline
\end{tabular}


En la Figura 8, el ensayo de CBR al 100\% muestra cuánto ha aumentado la resistencia del suelo con respecto al suelo natural. Un CBR el cual oscila entre $14,4 \%$ y $10,1 \%$ con la incorporación del $10 \%$ de VSO; un CBR con resultados entre $15,7 \%$ y $12,5 \%$ con la incorporación del $15 \%$ de VSO; un CBR con resultados entre $18,3 \%$ y $16,6 \%$ con el $20 \%$ de aglomerante $y$, por último, un CBR el cual oscila entre $20,5 \%$ y $17,4 \%$ con la incorporación del 25\%.

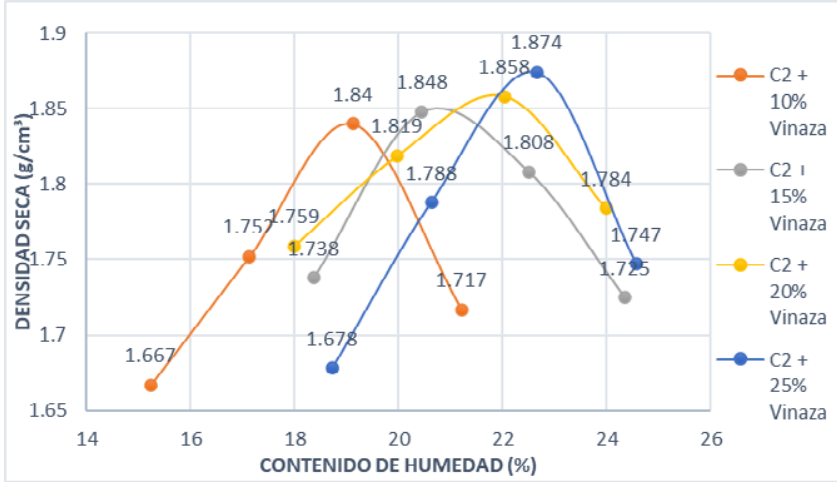

Figura 6. Resultados del ensayo de Proctor modificado según porcentajes de vinaza de Saccharum officinarum adicionados a las muestras de suelo

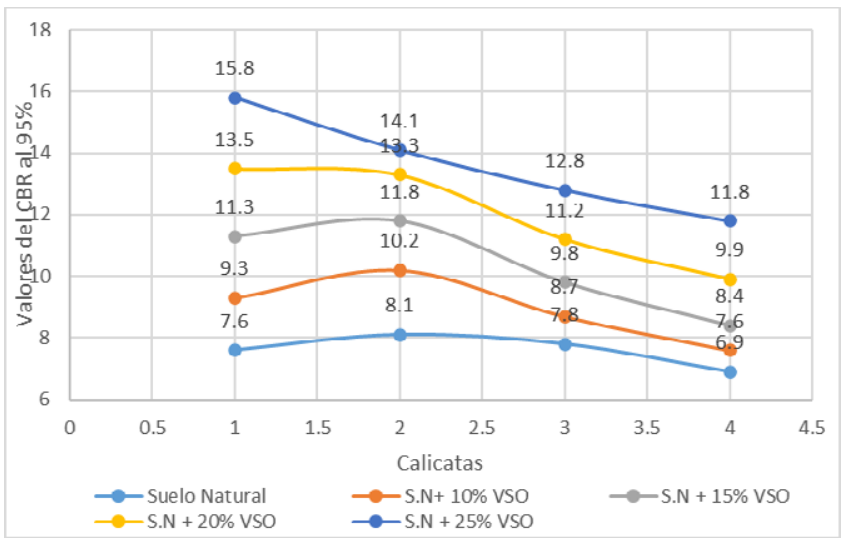

Figura 7. Resultados del ensayo de CBR al 95\% según porcentajes de vinaza de Saccharum officinarum adicionados a las muestras de suelo

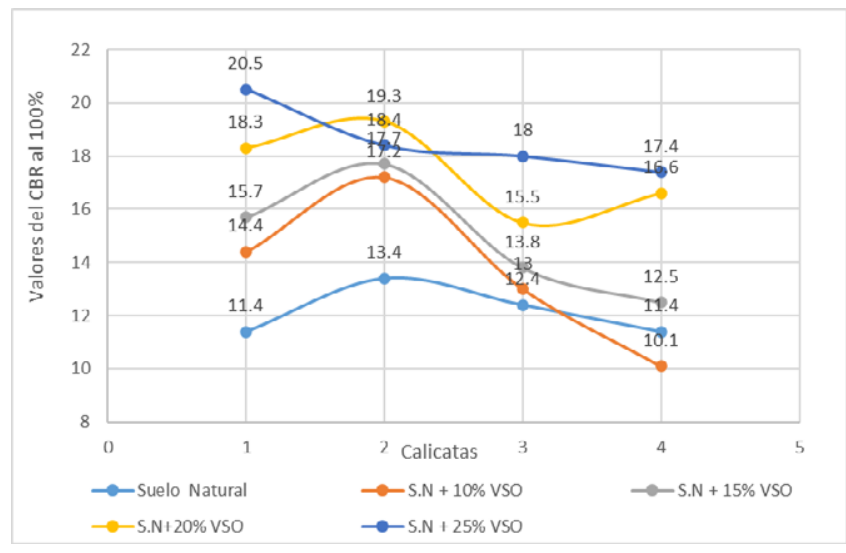

Figura 8. Resultados del ensayo de CBR al 100\% según porcentajes de vinaza de Saccharum officinarum adicionados a las muestras de suelo

Lozano y colaboradores (2015) realizaron la incorporación del 7\% del aglomerante a una muestra de suelo para realizar el ensayo de Proctor modificado, obteniendo como resultado una densidad máxima de $1,802 \mathrm{~g} / \mathrm{cm}^{3}$ y un óptimo contenido de humedad de 10,1\%. Así mismo, Cahuana (2018) realizó el ensayo de CBR al 95\% con la dosificación del $6 \%$ de vinaza, obteniendo un CBR de $43,40 \%$, indicando que es un CBR bueno según (Ministerio de Transportes y Comunicaciones, 2017). Ambas investigaciones desarrollan la misma metodología que esta investigación llevando a cabo el desarrollo de los mismos ensayos, como son Proctor modificado y CBR.

El Cuadro 4 plasma los valores del ensayo de valor de soporte (CBR) al $95 \%$ y $100 \%$ para determinar las propiedades de resistencia del suelo cohesivo mediante la incorporación de la vinaza de Saccharum officinarum en diferentes porcentajes (10\%, 15\%, 20\% y $25 \%$ ), dando mejores resultados la incorporación del $25 \%$, permitiendo que mejore las propiedades de resistencia del suelo estabilizado.

\section{Cuadro 4. Resultados del ensayo CBR 95\% y 100\% para el mejor porcentaje de dosificación}

\begin{tabular}{|c|c|c|c|}
\hline Dosificación de vinaza de Saccharum officinarum & Calicatas & CBR 95\% & CBR 100\% \\
\hline \multirow{2}{*}{$25 \%$} & C1 & 15,8 & 20,5 \\
\cline { 2 - 4 } & C2 & 14,1 & 18,4 \\
\cline { 2 - 4 } & C3 & 12,8 & 18,0 \\
\cline { 2 - 4 } & C4 & 11,8 & 17,4 \\
\hline
\end{tabular}


Musinguzi y Makomiju (2019) indicaron que después de realizar los ensayos de Proctor Modificado y CBR determinaron que con el $4 \%$ de incorporación de vinaza de Saccharum officinarum se alcanzó los mejores resultados. Esta investigación no guarda relación con lo estudiado; debido a que el $25 \%$ de incorporación de vinaza mejora continuamente las propiedades mecánicas del suelo cohesivo. Por otro lado, Cahuana (2018), Loaiza (2018) y Cordova (2018) expresan que el $25 \%$ de incorporación de vinaza de Saccharum officinarum dio los mejores resultados del suelo cohesivo estabilizado. La investigación en mención concuerda con lo desarrollado.

\section{Conclusiones}

Referente a las características geotécnicas, el suelo de la trocha carrozable en estudio se conforma por limos y arcillas inorgánicas de mediana plasticidad.

Sobre la máxima densidad seca, la incorporación de vinaza de Saccharum officinarum logra incrementarlo notoriamente siendo la aplicación del $25 \%$ de VSO la que obtuvo el mayor valor.

Los valores de aplicación de vinaza de Saccharum officinarum en la muestra de suelo natural con los diferentes porcentajes de incorporación oscilan entre $50 \mathrm{~g}$ y $125 \mathrm{~g}$.

El CBR del suelo estabilizado con el $25 \%$ de aglomerante oscilan entre $11,8 \%$ y $15,8 \%$, logrando determinar que el $25 \%$ de vinaza de Saccharum officinarum es el porcentaje óptimo para la estabilización de la trocha carrozable.

\section{Recomendaciones}

Se debe estudiar la aplicación de nuevos materiales reutilizados para la estabilización de suelos.

Se debe crear nuevas líneas de investigaciones relacionadas al uso de agentes estabilizantes de suelos cohesivos amigables con el ambiente.

\section{Agradecimientos}

En primer lugar, agradecer a Dios por brindarnos sabiduría para poder realizar esta investigación, a la Universidad Cesar Vallejo, a su laboratorio por haber permitido que se realicen los respectivos estudios de mecánica de suelos para así cumplir con el desarrollo de esta investigación.

\section{Referencias}

Alzate, A. (2015). Caracterización físico-química de una vinaza resultante de la producción de alcohol de una industria licorera, a partir del aprovechamiento de la caña de azúcar. Ingenierías USBMed, 6(2), 36-41. DOI: $10.21500 / 20275846.1729$

Loaiza, J. (2018). Mejoramiento de suelos GP con vinaza (Tesis de grado). Universidad Espíritu Santo, Ecuador.

Amunza, A. (2020). Stabilization of laterite soil for unpaved roads using molasses in butere and mumias sub counties (Tesis de grado). Jomo Kenyatta University of Agriculture and Technology, Kenia.

Becerra, J. (2019). Adición de miel de caña sobre el CBR del afirmado de la cantera El Gavilán, Cajamarca, 2017 (Tesis de grado). Universidad Privada del Norte, Perú.

Bhardwaj, A., Sharma, A., y Sharma, R. (2021). Stabilization of Clayey Soil Using Waste Foundry Sand and MolassesStabilization of Clayey Soil Using Waste Foundry Sand and Molasses. En Sustainable Development Through Engineering Innovations (pp. 641-649). Singapur:Springer Singapur. DOI:10.1007/978981-15-9554-7_57

Cahuana Cabanillas, F. (2018). Dosificación óptima del cloruro de calcio y melaza de caña para la estabilización de suelos en caminos vecinales no pavimentadas del distrito de Barranca 2016 (Tesis de grado). Universidad Nacional Santiago Antunez De Mayolo, Perú. 
Cordova Rubin, J. (2018). Utilización de la vinaza de caña de zúcar para estabilizar suelos cohesivos, Huancayo (Tesis de grado). Universidad Peruana Los Andes, Perú.

Kiran, Harsha, S., Raju, K. V. S. B., y Kumar, N. (2018). Experimental Study on Stabilization of Black Cotton Soil with Molasses and Arecanut Fibers. International Journal of Applied Engineering Research, 13(7), 219- 223.

Lozano, E., Ruiz, J., y Alfonso, J. (2015). Análisis del mejoramiento de un suelo de subrasante con un aditivo orgánico (Tesis de grado). Universidad Católica de Colombia, Colombia.

Ministerio de Transportes y Comunicaciones. (2017). Manual de ensayo de materiales. Lima, Lima, Perú. Recuperado de https://portal.mtc.gob. pe/transportes/caminos/normas_carreteras/ documentos/manuales/MANUALES\%20 DE\%20CARRETERAS\%202019/MC-06-16\%20 Manual\%20Ensayo\%20de\%20Materiales.pdf

Musinguzi, A., y Makomiju, C. (2019). A study on the performance of subgrade expansive soils modified with molasses and cement (Tesis de grado). Universidad de Makerere, Uganda.

Zúñiga Cerón, V., y Gandini Ayerbe, M. (2013). Caracterización ambiental de las vinazas de Residuos de caña de azúcar resultante de producción de etanol. Dyna, 80(177), 124-131.

Los contenidos de este documento, representan insumos dentro de un proceso de gestión del conocimiento que, por sí mismos, a priori, no constituyen una declaración de una normativa, procedimiento, criterio o herramienta oficial de acatamiento obligatorio en la gestión de proyectos de obra vial pública de Costa Rica, por parte del LanammeUCR. Cualquier posición oficial para Costa Rica sobre aspectos puntuales contemplados en este documento, se realizarán por los medios que corresponden, según los lineamientos de la Universidad de Costa Rica, de la Ley 8114 y su Reglamento al Art 6 (Decreto 37016 - MOPT). 